\title{
Materials fractal surface morphology used in the housing foundation exposed to aqueous media
}

\author{
R. R. Gallegos-Villela ${ }^{1}{ }^{*}$, J. A. Espuna-Mujica ${ }^{1}$, A. Palacio-Perez ${ }^{2}$, J. Gomez-Espinosa ${ }^{2}$, R.C. \\ Galindo-Lopez $^{3 *}$, Elena Izquierdo-Kulich ${ }^{4}$ \\ ${ }^{1}$ FADU Research Center. Architecture, Design and Urbanism Faculty. University Autonumus of \\ Tamaulipas (UAT). Circuito Interior S/N, Centro Universitario Sur. Tampico, Tamaulipas, Mexico, \\ *rafaela.gallegos@uat.edu.mx \\ ${ }^{2}$ CIPIA. Engineering Institute. Autonomous University of Mexico (UNAM), Mexico City, Mexico \\ ${ }^{3}$ Fluids Laboratory. Engineering Facullty. University Autonumus of Tamaulipas(UAT). Circuito Interior \\ S/N, Centro Universitario Sur. Tampico, Tamaulipas, Mexico*rgalindo@uat.edu.mx \\ ${ }^{4}$ Departaento de Química-Fïsica, Facultad de Química, Universidad de La Habana, La Habana, Cuba
}

\begin{abstract}
Foundations are a fundamental part of the structural framework in buildings, according to the final dimension and weight, it changes impact in loads transmition to the ground, and the structure design, as its mechanism of operation along with its useful life. However, the deterioration of the concrete or metal surface presented in the medium upon contact with the subsoil many times has not been considered. It depends on the characteristics and components of the site, as the solid surface, and chemical composition, of the structural elements. They can be altered by salty ingredients, modifying the structural properties with which it has been designed to support the building.

This research has been carried out to analyse the characteristics on the surfaces of the materials commonly used for the production of foundations in buildings (Steel, concrete, and wood), which are in principle underground, presenting various components.

Experimental and theoretical analysis of surfaces is carried out on elements of concrete, steel, and wood, exposed in an aqueous medium with and without salt, observing the microscopically change, fractal dimension, and rugosity modifications on the surface.

We found that the presence of saltwater tends to modify the fractal dimension and the specific surface area of concrete and steel majorly. For this case, the fractal dimension grows with corrosion and increase specific area too. We present a correlation for the three materials, which indicates that the presence of the salts significantly influences the roughness and porosity of the surface. These results may be used for resistance change with surface modification.
\end{abstract}

Key words: structural element, aqueous contact, surface modification, fractal dimension

\section{INTRODUCTION}

One of the fundamental parts in any type of building is the structure that composes and supports it [1] and, within it, the design and development of foundations, which can occur in superficial ones or shallow piles and derivation in micro piles [2].

Whatever the compelling case for the analysis and use of the building, the death, live, and service load transmitted continuously from the entire construction [3]. It will invariably be in contact with the subsoil in which different physicochemical agents are found [4]. Interaction of the foundations with fluid is possible in the case of a water reservoir with or without oil, that produces water that can migrate to the surface. Different factors degrade them, being different depending on material nature, geometry, a combination of elements, and the aqueous media [5].

The bases mainly use steel that can corrode if they are in contact with saline solutions [6], causing severe damage to the composition and resistance; a study of corrosion in these structures is essential because they can collapse under certain conditions such as seismic [6] or in constant contact with water surfaces. Underground we can find high and contaminated surfaces [7]. In the case of deep foundations, the study of corrosion is even more relevant because changes in these elements are not visible and sometimes surface treatments are necessary, as well as the element structural before placing in contact with salty aqueous [8]. Surface analysis studies focus on damage due to corrosion in acidic media or only in resistance [9], nevertheless, it is necessary a deeper análisis in Surface changes on time for a subsequent correlation with resistance degradation.

In México, foundations may be made of wood, with some standards specifications as N-CTR-CAR-1-02-011 [10], M-ctr - car-1-06-007 [11], among others. However, although there are some Surface effects as fungi growth or contact with 
specific saline solutions, which could improve conservation $[12,13]$, they do not specify these characteristics.

In this work, we present the study of the temporal behavior of the morphology in solid concrete, wood, and steel surfaces in contact with aqueous with and without salt media, under a controlled and recreated Lab environment. Taking into account that the study of materials is important in various areas of everyday life, such as dental [14].

\section{METHOD}

\section{1. creating synthetic water formation}

The synthetic deformation water was produced in a compound laboratory with Merck ACS salts: anhydrous calcium chloride $85 \mathrm{~g}$, magnesium chloride hexahydrate $33 \mathrm{~g}$, sodium chloride $89 \mathrm{~g}$, and sodium bicarbonate $39 \mathrm{~g}$, to generate every 4 liters of water formation, as shown in Figure 1. This salty water represents the one founded in water-oil reservoirs presented in Altamira, Tamaulipas, Mexico.

a.
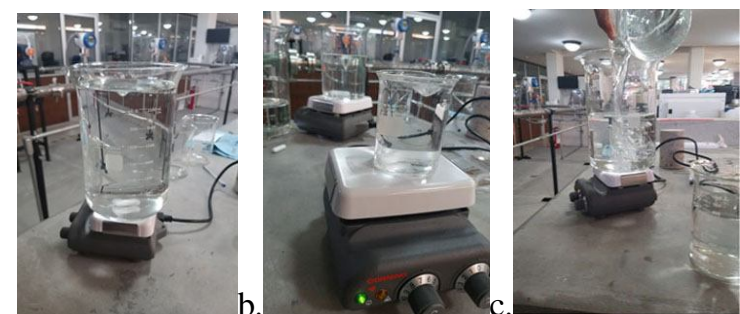

Figure 1: The mixture of the chemical components and the distilled water to form 4 liters of formation water, (a) stirrer of distilled water with magnesium chloride salt, (b), several beakers of $1000 \mathrm{ml}$ each, where distilled water is mixed with various salts, (c) placing distilled water in a glass of aforementioned to be stirred with the salts. Images captured by the authors.

This replication of reservoir water is with the intention of subjecting the solid elements to a highly saline environment and performing the morphological contrast of said surfaces by having direct and constant contact with them.

Three samples of materials were used: steel, concrete, and wood, with six repetitions each.

\subsection{Placing items in a controlled laboratory level environment}

Six samples of concrete, wood, and steel subjected to the controlled environment of synthetic water and a blank model in sweet-potable water, submerging $50 \%$ of the length of the samples to make the direct contact comparison against a surface not exposed to the aqueous médium (Figure 2).

\section{3. monitoring of specimens in aqueous contact}

During ten days, the samples in contact with the aqueous were monitored, obtaining physical dimensions, weight, and microscopic photographs in each of the samples, both in the area exposed directly to the aqueous medium and on the continuous surface with and without direct exposure; these images obtained are those that gave us the guide for the analysis of the fractal dimensions analyzed below. Figures 3 and 4 show the monitoring of the samples and the obtaining of microscopic images before obtaining the analyzed fractal dimension.

a.

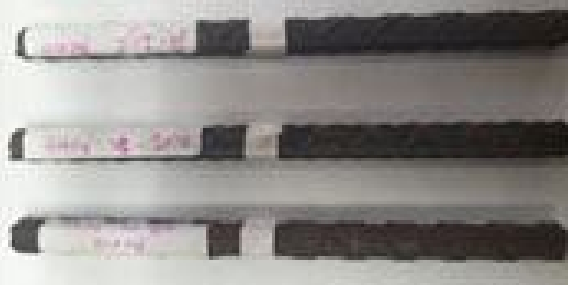

b.

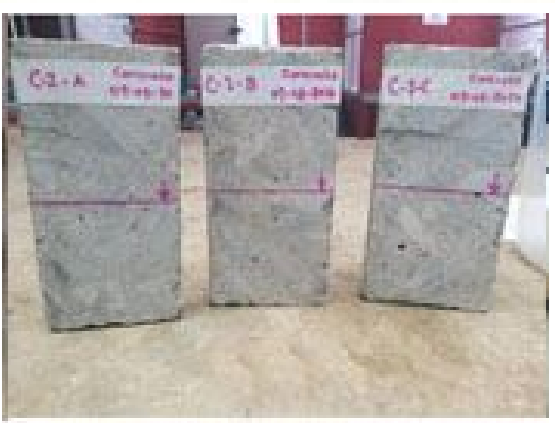

c.

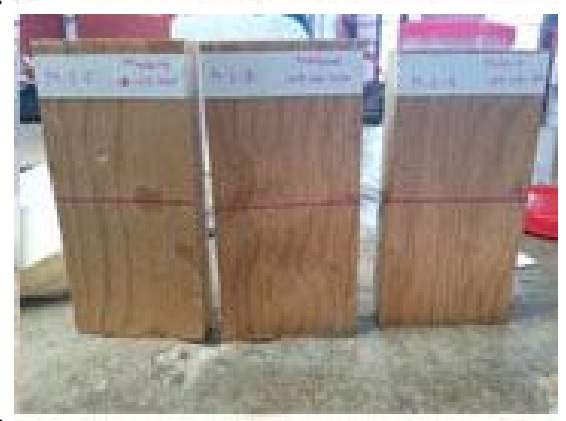

d.

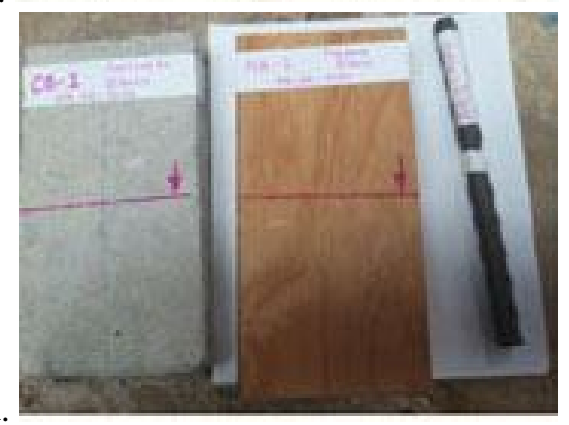

Figure 2: Specimens used, before aqueous contact. (a) 3/8" rod (b) concrete slats (c) wooden slats (d) concrete, wood, and steel specimens with the same dimensions to enter potable water. 


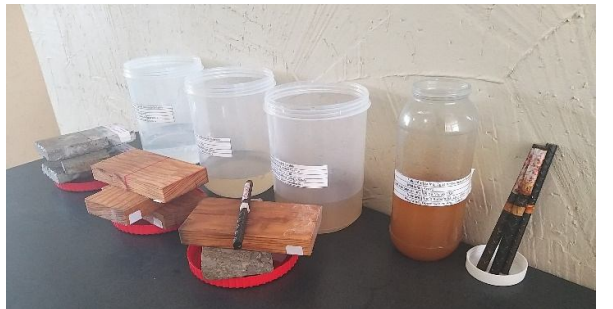

Figure 3: The image shows the schematic representation of daily monitoring of the specimens, which have previously been in aqueous contact within 24 hours before monitoring. Images captured by authors

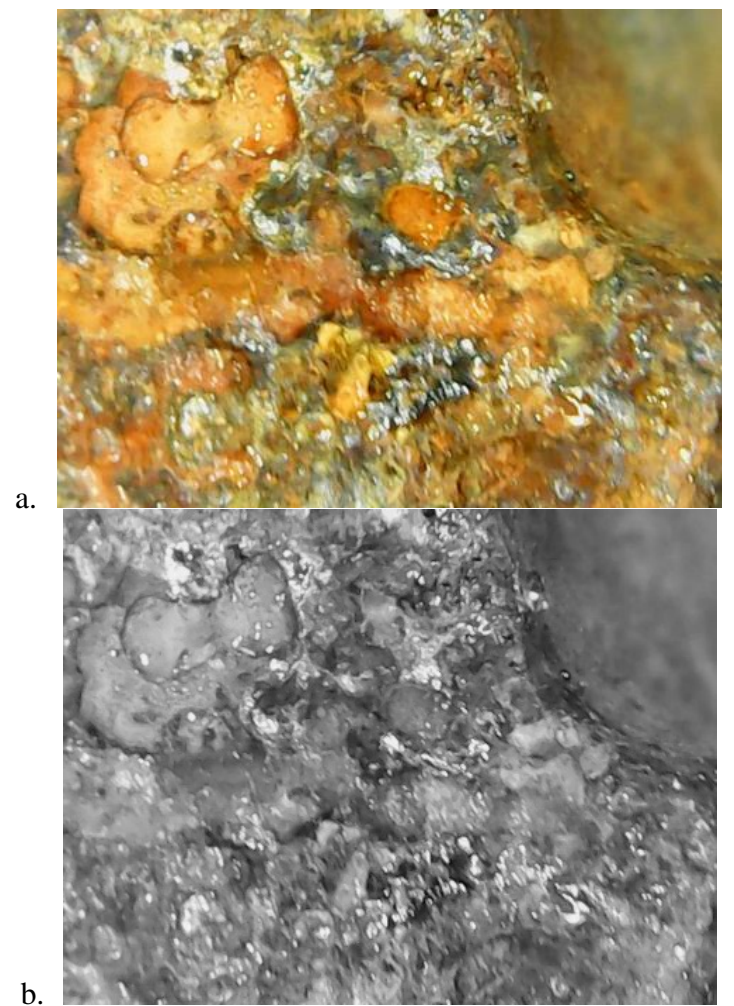

Figure 4: Image a shows the original state reached through daily microscopic monitoring of one of the steel samples in synthetic water, Image b. shows the

black and white treatment that is performed before the fractal dimension analysis. Images captured by the authors.

The micrographic analysis was carried out using ImageJ software according to the method presented in [15] and [16].

\section{RESULT AND DISCUSIÓN}

This paper aims to analyze how the morphology of a surface material used in construction changes when it is in contact with an aqueous medium that facilitates the appearance of processes of deposition and detachment of substances that affect the roughness of the surface.

Photographs were taken of the surface of three materials, steel, concrete, and cement, which are in contact with the water and the synthetic reservoir water, respectively, and it is also essential to analyze how the composition of the water influences in the effects observing on the surface. These photographs were taken every 24 hours ( 1 day) for ten consecutive days. Some representative images are shown in Figure 5.

Each photograph was processed using the ImageJ program, where the height difference of the interface, identified with the intensity of the pixels, and the fractal dimension of the curve representing the behavior of the pixel intensity (pixel height) was determined concerning the spatial position. For each case, we processed four images.

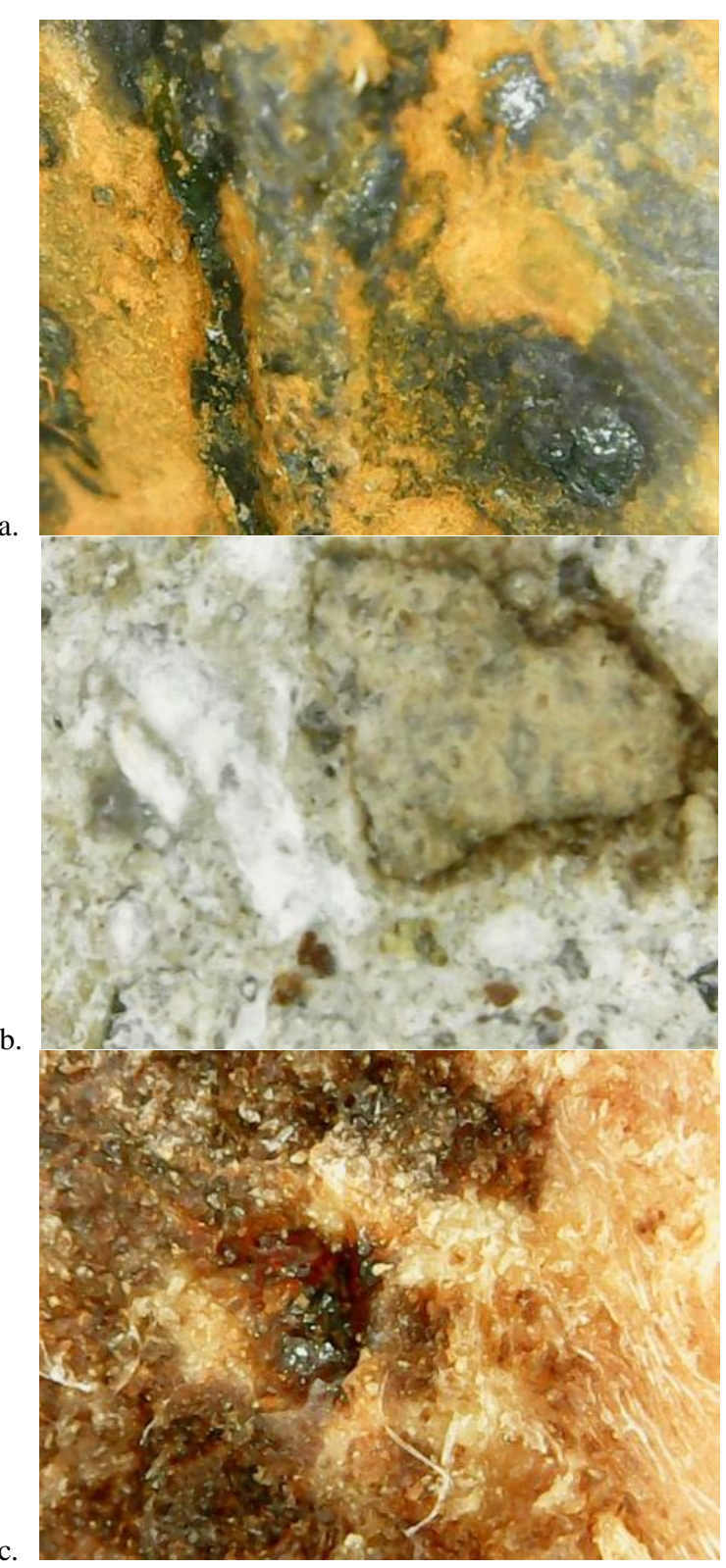

igure 5. Several images of the monitoring of surface microscopes of the samples. (a) bar surface on the ninth day of exposure to formation water, (b) surface of the concrete sample on the ninth day of aqueous exposure, (c) wooden surface on the ninth day of aqueous exposure. Images captured by the authors.

We quantified the surface roughness through two fundamental parameters, the fractal dimension of the surface, obtained by adding the fractal dimensions of the two images 
R. R. Gallegos-Villela et al.,International Journal of Emerging Trends in Engineering Research, 8(7), July 2020, 3525 - 3529

analyzed, and the specific fractal area as, f, which estimated through the relationship:

$$
a_{n, f}=\frac{1}{\Gamma\left(1+f_{x}\right) \Gamma\left(1+f_{y}\right)}\left(\frac{h_{\max }}{h_{\min }}\right)^{f-f-2} \frac{\mathrm{m}^{2}}{\mathrm{~m}^{2}}
$$

Being $h_{\max }$ and $h_{\min }$ the maximum and minimum values of the interface height, $\mathrm{fx}$, and $\mathrm{f}$ and the values of fractal dimension observed in the two samples corresponding to each case. In this sense, a more significant increase in the fractal dimension and the specific fractal area implies a more significant effect of the environment on it.

Specific fractal area changes itself according to the surface porosity increase in the time. On the other hand, substances deposition, that may eventually react, causing composition damage, growth with material porosity. In this case, biological contamination may occur, developing biofilms.

When conducting the steel study with drinking water, no significant differences were found in the samples. The fractal dimension of the surface had an average value of $1.2079 \pm$ 0.0501 for steel case, $1.2109 \pm 0.0301$ for concrete and 1.2979 \pm 0.0501 for wood.

The fractal dimension of the surface had an average value of 1.2379 \pm 0.0310 ; however, in the case of steel, and for concrete, no significant variations were foreseen.

Figure 6 show the results for the fractal determination in the case of salty water or synthetic reservoir water.

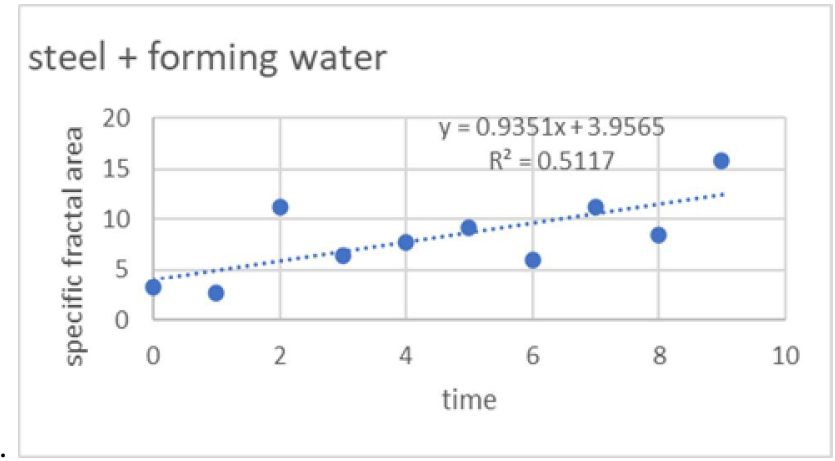

a.

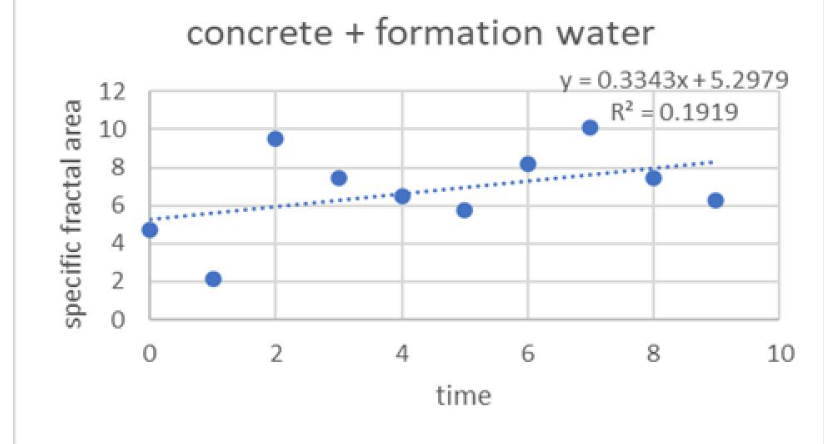

b.

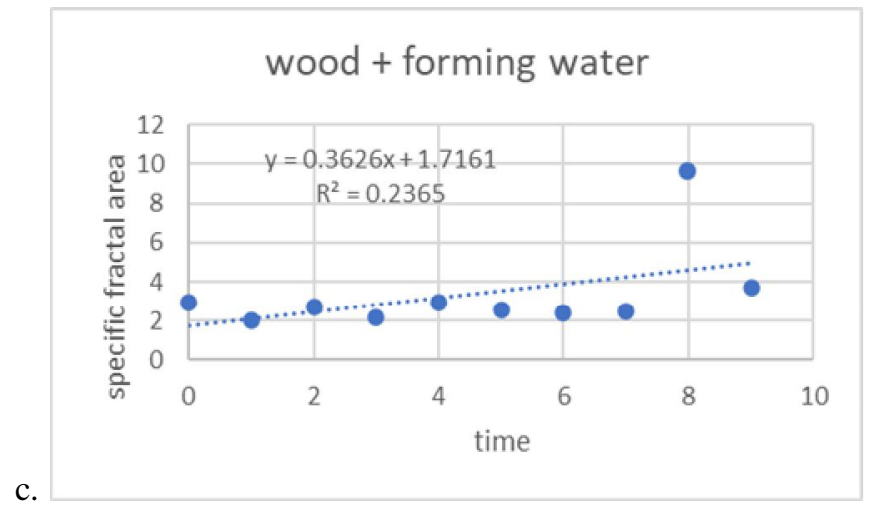

Figure 6: The behavior of the specific fractal area, for a. Steel b. Concrete, and c. wood

The experimental results obtained suggest that the presence of water tends in general to modify the fractal dimension and the specific surface area of the three materials analyzed. In this context, when considering water, the material where there was a slight tendency to increase in the fractal dimension was wood, while in the case of cement and steel, practically no correlation was observed. In the case of reservoir water, a correlation was obtained for the three materials, which indicates that the presence of the salts that are present in the formation water significantly influences the roughness and porosity of the surface.

For this experimental analysis, the period inspected before the specimens exposed to the aqueous medium was brief in comparison to an estimated lifetime of a building; however, a change in the surfaces was perceived changing the roughness and porosity, which for a long and constant exposure can detonate a physical variable in terms of its dimensions and therefore in the mechanical properties of said structural elements.

\section{CONCLUSION}

After carrying out constant monitoring of the samples in contact with the potable water and the water from the synthetic formation, presenting the analysis of the micrographs obtained on each of their surfaces, we can conclude that the materials in contact with the fluid are not affected to a great extent, in the three case studies, steel, concrete and wood, the one with the largest fractal dimension in terms of the porosity manifested on its surface is the sample of wood, in direct contact with the water. On the other hand, in the examples of the various materials analysed, in touch with the synthetic-forming water fluid, they manifested changes in the surfaces through the roughness and porosity of the element, indicating that the presence of the salts found in the formation the water influences in a significant way the initial composition of these elements. 
R. R. Gallegos-Villela et al.,International Journal of Emerging Trends in Engineering Research, 8(7), July 2020, 3525 - 3529

In addition to the previous, we denoted that through the constant monitoring of the specimens. We observed mold on the dry surfaces of wood samples that were in contact with salty water. For the concrete and steel cases, we see that deterioration can be sized by fractal dimension.

The fractal dimension of the surface profile was $1.2079 \pm 0.0501$ for steel case, $1.2109 \pm 0.0301$ for concrete but increasing to $1.2979 \pm 0.0501$ for wood. From different results, for water with and without salts, in the case of steel, and concrete, no significant variations were foreseen. In the case of salty water with the samples, specific area increase in a short time.

In further work, it is necessary to increase the experimental time exposition.

\section{REFERENCES}

[1]. Lanzas Sánchez, A., \& Antón i Vázquez, D. (2018). Diseño de instalaciones y estructura de un edificio de 40 viviendas (Bachelor's thesis, Universitat Politècnica de Catalunya).

[2]. Felipe, G. M. (2017). Sílabo de Ingeniería de cimentaciones.

[3]. Barrantes Mann, L. A. J. (2018). Tecnología para cimentaciones por pilotaje en proyectos de ingeniería civil.

[4]. Tejada Rodríguez, F. L. (2019). Efectos de interacción suelo-estructura en la respuesta sísmica de edificios altos (Master's thesis, Universitat Politècnica de Catalunya).

[5]. Fernandes, B., Christ, R., Quinino, U., \& Tutikian, B. (2016). Análisis de concretos con no conformidades: efectos de larga duración. Revista ALCONPAT, 6(3), 261-270. https://doi.org/10.21041/ra.v6i3.154

[6]. Kennedy, C., TrustGod, J. A., \& Okpara, J. C. (2019). Reinforcing steel mechanical properties influence on bond strength of corroded and coated members in concrete structures. GSJ, 7(9).

[7]. Rojas Ticona, Y. H. (2016). Patologías en Cimentaciones Superficiales Generados por el Agua y Rellenos Contaminados en la Zona de Salcedo-Puno.

[8]. Gao, J., Tang, D., \& Ashraf, M. A. (2019). Analysis of Deep Foundation Treatment of Soft Soil Under Strong Corrosion Conditions. Nature Environment \& Pollution Technology, 18(5).

[9]. Wang, S. G., Han, H. B., Sun, M., \& Long, K. (2013). X-Ray Photoelectron Spectroscopy Characterization for the Electrochemical Corrosion of Bulk Nanocrystalline 304 Stainless
Steel in Hydrochloric Acid. Recent Patents on Corrosion Science, 3(2), 164-170.

[10]. Norma Mexicana N-CTR-CAR-1-02-01, construcción, conceptos de obra, estructuras, preservación de madera, Secretaria de Comunicación y Transporte (SCT), Diciembre 2000.

[11]. Norma Mexicana M-ctr- car-1-06-007, construcción, conceptos de obras, cimentaciones, pilotes colados en sitio, Secretaria de comunicaciones y transporte (SCT), Diciembre 2001.

[12]. Brischke, C., \& Alfredsen, G. (2020). Wood-water relationships and their role for wood susceptibility to fungal decay. Applied Microbiology and Biotechnology, 1-15. https://doi.org/10.1007/s00253-020-10479-1

[13]. Luo, X., Cheng, J., Xiang, P., \& Long, H. (2020). Seismic behavior of corroded reinforced concrete column joints under low-cyclic repeated loading. Archives of Civil and Mechanical Engineering, 20(2), 1-20. https://doi.org/10.1007/s43452-020-00043-z

[14]. Angham, H., Hayder, M. A., \& Ahmed, H. (2019). Structural, electronic, optical properties and antibacterial application of novel (PMMA-Al2O3-Ag) nanocomposites for dental industries applications. Int. J. Emerg. Trends Eng. Res. 7 [8] 104-123 https://doi.org/10.30534/ijeter/2019/04782019

[15]. Suarez-Dominguez, E. J., Perez-Rivao, A., Sanchez-Medrano, M. T., Perez-Sanchez, J. F., \& Izquierdo-Kulich, E. (2020). Mesoscopic model for the surface fractal dimension estimation of solid-solid and gas-solid dispersed systems. Surfaces and Interfaces, 18, 100407. https://doi.org/10.1016/j.surfin.2019.100407

[16]. Valle-Chavarria, L. G., Berumen-Rodriguez, C. E., \& Suarez-Dominguez, E. J. (2020). A Study of the Formal Architectural-Sculptural Characteristics of El Tajin. Conservation Science in Cultural Heritage, 19(1), 143-156. 